\title{
En hommage à Angelos Kalogeropoulos
}

\section{Angelos Kalogeropoulos nous a quittés, foudroyé par une leucémie}

La foule qui assistait à ses obsèques, ce mardi ensoleillé d'avril, en disait long sur l'amour, l'amitié, l'estime qu'il avait suscités autour de lui. Venu en France du temps où la Grèce souffrait de dictature, il a participé, avec Gérard, Alain, Normand, Ali, Hanafy et Sylvie, à l'aventure de l'analyse génétique des mécanismes de la recombinaison méiotique chez Ascobolus immersus, ce champignon exotique, fantaisiste et attachant introduit par Georges Rizet. Avec Pierre Thuriaux, pendant et après le séjour sabbatique de Pierre dans notre laboratoire, il développa une analyse mathématique des paramètres de la conversion génique dont certaines prédictions ont été vérifiées depuis. C'est au cours de ce travail qu'il a appris à bâtir des programmes informatiques. Cela l'a conduit par la suite à développer un projet qui exploitait les informations fournies par le séquençage du génome de la «levure» afin, notamment, de mieux caractériser les gènes et leurs séquences régulatrices. La dynamique qu'il avait créée lui a permis de réunir rapidement autour de lui une équipe de "bioinformaticiens du génome ", de nouer plusieurs collaborations à

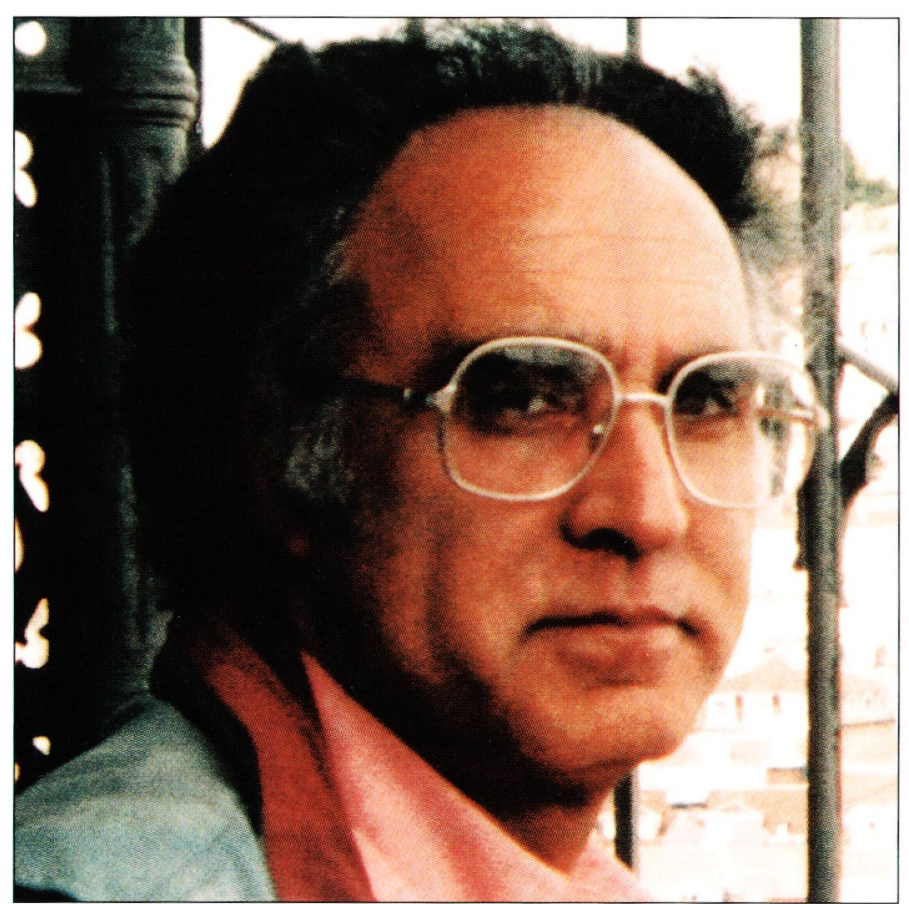

Il a été pendant de nombreuses années un pilier du DEA de Génétique Cellulaire et Moléculaire, dispensant aux étudiants ses conseils et ses remontrances bienveillantes, s'assurant de leur progression pendant et après leur thèse, les défendant avec passion.

Angelos avait connu, sous la dictature des colonels grecs, la bétise et la haine. Il s'était engagé politiquement pour servir un idéal de générosité, pour bâtir un monde d'égalité et de fraternité. Il croyait dans la raison. Pour lui la science et la politique découlaient d'une même

de se faire apprécier dans ce monde spécialisé.

Angelos était de ceux qui, ne refusant jamais un service, étaient continuellement sollicités. Ses compétences en informatique, un atout précieux pour la communauté, lui ont valu de consacrer ces dernières années beaucoup de temps à aider les autres. La maladie qui l'a emporté a été classée "maladie professionnelle». Il est de ceux qui ont pris des risques en utilisant pour effectuer des mutagenèses des produits dont on n'imaginait pas encore à quel point ils pouvaient être nocifs. Lorsque la nature des risques s'est précisée, il a été un des premiers à les mesurer et à les prendre en compte, assurant la mise en place, puis la responsabilité d'utilisation de la première pièce à mutagenèse du bâtiment 400. rationalité. Il ne croyait pas au ciel, mais il était passionné par le mystère de l'Univers et la quête des astrophysiciens. Il croyait qu'un jour, on pourrait construire un paradis sur la terre. Une société juste et fraternelle où la connaissance serait sacrée.

Pour chacun de ceux qui l'ont connu, Angelos est toujours là. Je l'entends rire. Un sourire moqueur et chaleureux.

\footnotetext{
Jean-Luc Rossignol

Institut de génétique et microbiologie, Université Paris-Sud, 91405 Orsay Cedex, France.
} 


\section{Angelos: I'approche bio-informatique}

Les premiers travaux d'Angelos ont été consacrés à l'analyse génétique de la recombinaison. A partir des données acquises, il s'est associé à $\mathrm{P}$. Thuriaux pour élaborer un modèle numérique. Ce faisant, il dut apprendre à programmer un ordinateur et découvrit ainsi le monde de la bio-informatique.

Le séquençage du génome de levure commençait et beaucoup étaient sceptiques sur ces approches systématiques. Angelos, qui avait consacré tout son travail scientifique à un petit nombre de gènes, vit tout l'intérêt qu'il y aurait à explorer et analyser ces treize millions de paires de bases. Cette analyse ouvrait la possibilité de survoler le génome entier, d'en déceler les points remarquables et d'établir des prédictions.

Sa démarche suivit alors deux axes: l'analyse linguistique des séquences, selon le schéma d'E.N. Trifonov (qui allait devenir son ami), et l'analyse des signaux de régulation de la transcription. Ainsi, il a démontré que le chromosome III de la levure est constitué de «mots» de 4 ou 5 nucléotides, démontrant que l'analyse linguistique, jusque-là vérifiée sur des séquences concaténées, était validée par l'étude d'un chromosome entier d'Eucaryote. D'un autre côté, il a élaboré un logiciel détectant automatiquement les introns et certaines UAS : ce logiciel est couramment utilisé par la communauté des levuristes.

Rapidement, il allait attirer des émules et former un petit groupe dynamique. Son approche allait vite être reconnue par la communauté internationale: il fut l'organisateur d'un colloque in silico à Orsay (1993) auquel participèrent les meilleurs spécialistes mondiaux.

Par ailleurs, il se méfiera tout le temps du danger majeur encouru par tout théoricien : la déconnexion du travail expérimental. Pour y pallier, il gardera un contact étroit avec ses amis généticiens moléculaires de l'IGM pour que ses prédictions ou celles de son équipe soient, autant que possible, vérifiées expérimentalement.

Enseignant-chercheur, il était capable de transmettre aux étudiants les domaines émergeants de la biologie. Il était, à Orsay, un animateur essentiel de l'enseignement de la Bio-informatique à tous les niveaux.
Il nous a quitté trop tôt. A nous de poursuivre dans la voie qu'il a ouverte. Si vous souhaitez marquer votre attachement à la mémoire d'Angelos, il vous est possible d'adresser un don au compte bancaire dont les coordonnées vous sont données ci-dessous.

Les fonds recueillis seront destinés au Service d'Hygiène et de Sécurité.

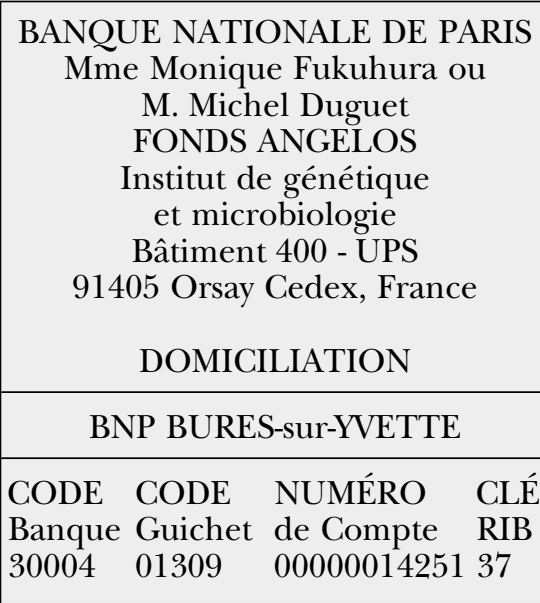
M. Bolotin-Fukuhara
M. Jacquet
M. Termier

\section{INFORMATIONS SFG}

\section{Première conférence Louis Pasteur sur les maladies infectieuses "Génétique de la sensibilité aux maladies infectieuses" Institut Pasteur, Paris, France - 21-23 octobre 1996}

Dans un proche avenir, il sera possible de cribler l'ensemble du génome humain à la recherche de gènes qui contrôlent la sensibilité à une infection et la pathogénie de la maladie associée. Cette congérence rassemblera des généticiens, des immunologistes et des microbiologistes qui discuteront des conséquences de ces développements pour la médecine.

\section{Programme}

Conférence inaugurale: C. Weissmann (Zurich, Suisse) Maladies multifactorielles et multigéniques chez l'homme L. Abel (Paris, France), W. Dietrich (Cambridge, États-Unis), G.M.Lathrop (Oxford, Royaume-Uni), J. Weissenbach (Paris, France)

Polymorphisme génétique et déficits immunitaires

A. Fischer (Paris, France), B. Mach (Genève, Suisse), F.S. Rosen (Boston, États-Unis), O.N. Witte (Los Angeles, ÉtatsUnis)

Paludisme et autres maladies parasitaires

A. Dessein (Marseille, France), A.V.S. Hill (Oxford, RoyaumeUni), D. Kwiatkowski (Oxford, Royaume-Uni), L.H. Miller (Bethesda, États-Unis), D.J. Weatherall (Oxford, Royaume-Uni) Tuberculose et autres maladies bactériennes
J.N. Blackwell (Cambridge, Royaume-Uni), P. Gros (Montréal, Canada), S.H.E. Kaufmann (Ulm, Allemagne), E. Skamene (Montréal, Canada)

Maladies virales

M. Brahic (Paris, France), B.W. Chesebro (Hamilton, ÉtatsUnis), O. Haller (Freiburg, Allemagne), D. Kabat (Portland, États-Unis), G.R. Shellam (Nedlands, Australie)

Conférence de clôture

R.M. Anderson (Oxford, Royaume-Uni)

Organisateurs : Louis H. Miller (Président), Michel Brahic, Marc Fellous, Emil Skamene

Pour tout renseignement, s'adresser à :

Bénédicte Écoutin - Centre d'Information Scientifique, Institut Pasteur, 28, rue du Docteur-Roux, 75724 Paris Cedex 15, France. Tél. : 1-40.61.34.53

Télécopie : $1-40.61 .34 .05$

E-mail : becoutin @ pasteur.fr 